\title{
On the asymmetry of velocity oscillation amplitude in bipolar active regions
}

\author{
F. Giannattasio ${ }^{1}$, M. Stangalini ${ }^{1,2}$, D. Del Moro ${ }^{1,2}$, and F. Berrilli ${ }^{1}$ \\ 1 Dipartimento di Fisica, Università di Roma "Tor Vergata", via della Ricerca Scientifica 1, 00133 Rome, Italy \\ e-mail: Fabio.Giannattasio@roma2.infn.it \\ 2 Max Planck Institute for Solar System Research, Max-Planck-Str. 2, 37191 Katlenburg-Lindau, Germany \\ Received 25 July 2012 / Accepted 10 December 2012
}

\section{ABSTRACT}

\begin{abstract}
The velocity field in the lower solar atmosphere undergoes strong interactions with magnetic fields. Many authors have pointed out that power is reduced by a factor between two and three within magnetic regions, depending on frequency, depth, the radius, and the magnetic strength of the flux tube. Many mechanisms have been proposed to explain the observations. In this work, Solar Dynamics Observatory (SDO) dopplergrams and magnetograms of 12 bipolar active regions ( $\beta$ ARs) at a 45-s cadence are used to investigate the relation between velocity fluctuations and magnetic fields. We show that there is an asymmetry within $\beta$ ARs, with the velocity oscillation amplitude being more suppressed in the leading polarities than in the trailing polarities. Also, the strongest magnetic fields do not completely suppress the five-minute oscillation amplitude, even in the spot's innermost umbrae.
\end{abstract}

Key words. Sun: activity - Sun: oscillations

\section{Introduction}

The study of the interaction between waves and magnetic fields plays an important role in the investigation of the mechanisms responsible for solar corona heating. It is well established that the amplitude of solar five-minute oscillations in the photosphere is reduced by a factor of two to three within active regions (ARs; Leighton et al. 1962; Woods \& Cram 1981; Lites et al. 1982; Abdelatif et al. 1986; Braun et al. 1987; Tarbell et al. 1988; Braun et al. 1988; Title et al. 1992; Hindman \& Brown 1998). The reduction is observed to depend on frequency, depth, and on the physical parameters that describe the flux tubes, i.e., their radius and magnetic strength (Brown et al. 1992; Hindman \& Brown 1998; Gordovskyy \& Jain 2008). In addition, this behavior has been found in small magnetic field concentrations (Roberts \& Webb 1978; Spruit 1981; Bogdan et al. 1996; Cally \& Bogdan 1997; Hindman \& Jain 2008, 2012; Jain et al. 2009; Pradeep Chitta et al. 2012).

It is also known that at higher frequencies, above the acoustic cutoff frequency in the low photosphere, the velocity oscillation amplitude is enhanced at the edges of ARs (Braun et al. 1992; Brown et al. 1992; Toner \& Labonte 1993). Furthermore, an enhancement of power in the three-minute band has recently been observed in the inner umbra of a pore (Stangalini et al. 2012).

Several mechanisms have been proposed to explain the observations (Hindman et al. 1997):

1) Intrinsic power inhibition due to local convection suppression. By using numerical simulations, Parchevsky \& Kosovichev (2007) found that the reduction of wave excitation in a sunspot can account for up to $50 \%$ of the power deficit.

2) Partial p-mode absorption (Braun et al. 1987; Bogdan et al. 1993; Cally 1995; Cally \& Andries 2010). Spruit \& Bogdan (1992) showed that wave absorption by sunspots can be interpreted in terms of $\mathrm{p}$-mode conversion between the oscillations and magnetohydrodynamics (MHD) waves. Both models (Cally \& Bogdan 1993) and simulations (Cameron et al. 2008) have shown that mode conversion is able to remove a significant amount of energy from the incident helioseismic wave with an efficiency that depends on the angle of the magnetic field from the vertical, with a maximum at about $30^{\circ}$ (Cally et al. 2003; Crouch \& Cally 2003).

3) Opacity effects. Within a magnetic region, the line-of-sight optical depth experiences a depression (Wilson depression). Due to increasing density, the amplitude of the velocity fluctuations decreases with depth.

4) Alteration of the p-mode eigenfunctions by the magnetic field (Jain et al. 1996; Hindman et al. 1997).

We note that the majority of studies on the interaction between solar oscillations and magnetic field have focused on the use of models and simulations.

Because of the dependence of velocity amplitude on magnetic strength, the most noticeable effects are observed within ARs, where the strongest magnetic fields are found. Among them, bipolar active regions (hereafter $\beta$ ARs) are particularly interesting, showing asymmetries in their morphology and physical properties, as several works have already pointed out (see, e.g., Bray \& Loughhead 1979; Balthasar \& Woehl 1980; Ternullo et al. 1981; Zwaan 1985; van Driel-Gesztelyi \& Petrovay 1990; Petrovay et al. 1990; Fan et al. 1993). MorenoInsertis et al. (1994) conclude that morphological asymmetries are due to the different inclination with which the polarities of $\beta$ ARs emerge.

Using simulations, Fan et al. (1993) have studied area asymmetries, predicting that at the same depth the leading polarity must have a strength about twice as large as the trailing polarity. This result, which is a consequence of Coriolis force action on the magnetic structure, is able to account for the trailing spot fragmentation, its lower flux magnitude, and its shorter lifetime. 
Recently, Michelson Doppler Imager (MDI) observations of 138 bipolar magnetic regions have quantitatively shown that the areas of leading polarities are typically smaller than those of trailing polarities (Yamamoto 2012). This area asymmetry could be produced by the Coriolis force during a flux tube's rising motion in the solar convection zone.

In this work, we investigated the velocity oscillation amplitude reduction by the magnetic field within $\beta$ ARs. We analyzed $12 \beta$ ARs from SDO-HMI (Helioseismic and Magnetic Imager) data, both in the northern $(\mathrm{N})$ and southern $(\mathrm{S})$ hemispheres, and found that the leading polarity systematically has a greater reduction in amplitude than the trailing. In addition, we studied the amplitude reduction as a function of the field inclination for AR11166.

\section{SDO-HMI data}

Our dataset consists of 12 SDO-HMI magnetograms and dopplergrams pairs with 1 arcsec spatial resolution (Scherrer et al. 2012; Schou et al. 2012). These were acquired with a 45-s cadence, which set the Nyquist frequency. Each $\beta \mathrm{AR}$ dataset is three hours long, which set the lower cut-off at $\sim 10^{-4} \mathrm{~s}^{-1}$. Observation times covered the interval from 2011 March 8 to 2012 January 3 (see Table 1).

We selected eight isolated bipolar regions in the $\mathrm{N}$ hemisphere and four in the $\mathrm{S}$ hemisphere. In order to limit the effects due to the inclination with respect to the line of sight, we selected $\beta$ ARs as close as possible to the disk center. The rectangular area enclosing the $\beta$ ARs was selected such that it included all magnetic features with strength above $500 \mathrm{G}$, with the the minimum and maximum allowed dimensions of the enclosing rectangle being 500 and 700 arcsec, respectively. Figure 1 shows the mean magnetograms of two selected $\beta$ ARs: AR 11166 in the $\mathrm{N}$ hemisphere and AR 11316 in the $S$ hemisphere. In both panels, the leading polarity is on the right and is negative for the $\mathrm{N} \beta \mathrm{ARs}$, positive for the $\mathrm{S} \beta$ ARs. Table 1 shows the list of $\beta$ ARs analyzed in this work.

\section{Method and analysis}

The selected regions around each $\beta \mathrm{AR}$ were co-registered using a fast Fourier transform (FFT) technique that ensures subpixel accuracy. Inspection of the co-registered data shows that the strong magnetic structures remain in the same position during the entire duration of the acquisition. The amplitude of velocity oscillations was estimated pixel by pixel through their integrated spectrum. Specifically, we selected a spectral window $\Delta v$ centered at $3 \mathrm{mHz}$ (five-minute band) with a width of $1 \mathrm{mHz}$ and then integrated the FFT amplitude spectrum $A_{v}$ in that frequency range:

$A_{x y}=\int_{\Delta v} A_{v} \mathrm{~d} v$

We then divided the data into bins of magnetic field, each $25 \mathrm{G}$ wide, and averaged the amplitude of velocity oscillations in each bin, thus obtaining $A_{B}=\left\langle A_{x y}\right\rangle_{B}$. We consider only pixels with $|\mathbf{B}|>25 \mathrm{G}$. Since all the amplitude distributions within the respective magnetic bin have a Gaussian-like shape (see inset in Fig. 2b), we use $\sigma / \sqrt{n}$ as error on $A_{B}$, where $\sigma$ is the standard deviation and $n$ are the counts in each bin.

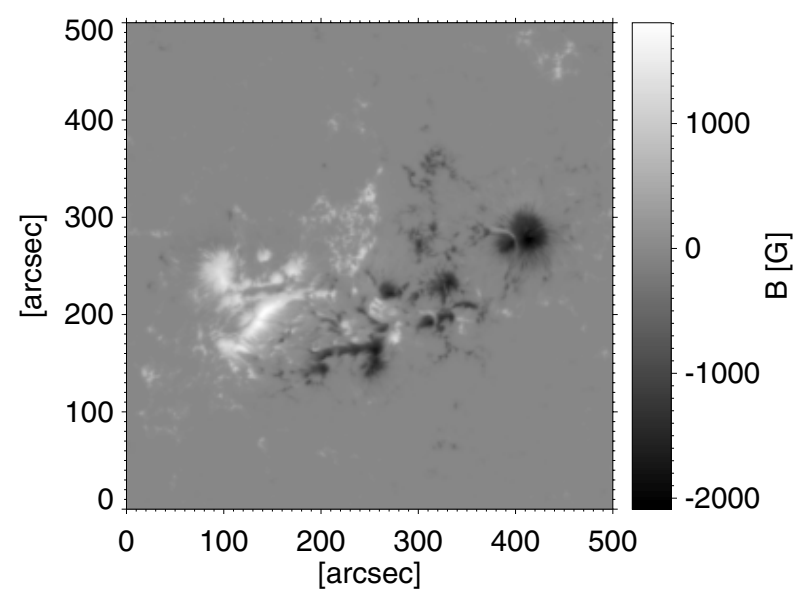

(a) AR 11166

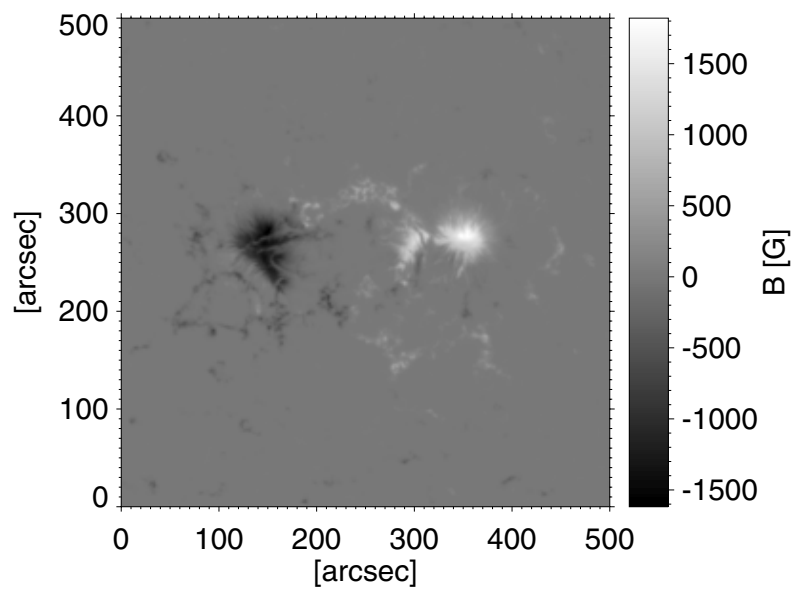

(b) AR 11316

Fig. 1. SDO-HMI mean magnetograms of a) AR 11166 b) AR 11316. See Table 1 for more details.

\section{Results and discussion}

\subsection{Oscillation amplitude vs. magnetic field strength}

In Fig. 2 we show the integrated amplitude $A_{x y}$ scatter plot (black dots) and $A_{B}$ for each magnetic bin (red line) for the $\beta$ ARs shown in Fig. 1. Hereafter, we refer to the $A_{B}$ plot as the amplitude profile. The amplitude shows a decreasing trend up to a plateau, which is almost always reached in the range $0.5-1 \mathrm{kG}$ (as in the case of Fig. 2b). This feature suggests that the amplitude is independent of the magnetic field strength above a threshold value, which is different for each $\beta \mathrm{AR}$ and each polarity in the same $\beta$ AR.

For every polarity present in the $12 \beta \mathrm{ARs}$ analyzed, we computed the oscillation amplitude reduction as the ratio $d=$ $A_{1000} / A_{25}$, where $A_{1000}$ and $A_{25}$ are, respectively, the mean oscillation velocity around $B=1000 \mathrm{G}$, and $B=25 \mathrm{G}$, the minimum observable strength. Averaging the $d$ values over all the $\beta$ ARs, we obtained $\bar{d}=0.54 \pm 0.06$, which agrees with the oscillation amplitude reductions in magnetic environments quoted in the literature (e.g., Woods \& Cram 1981; Lites et al. 1982; Braun et al. 1987; Tarbell et al. 1988).

The plateau in the amplitude profiles for high $B$ values is intriguing. By visual inspection, we verified that it takes place almost exclusively in the $\beta \mathrm{AR}$ spot umbrae, as can be expected, given the high magnetic field values. We can exclude an instrumental effect, since the magnetic strength threshold changes 
F. Giannattasio et al.: On the asymmetry of velocity oscillation amplitude in bipolar active regions

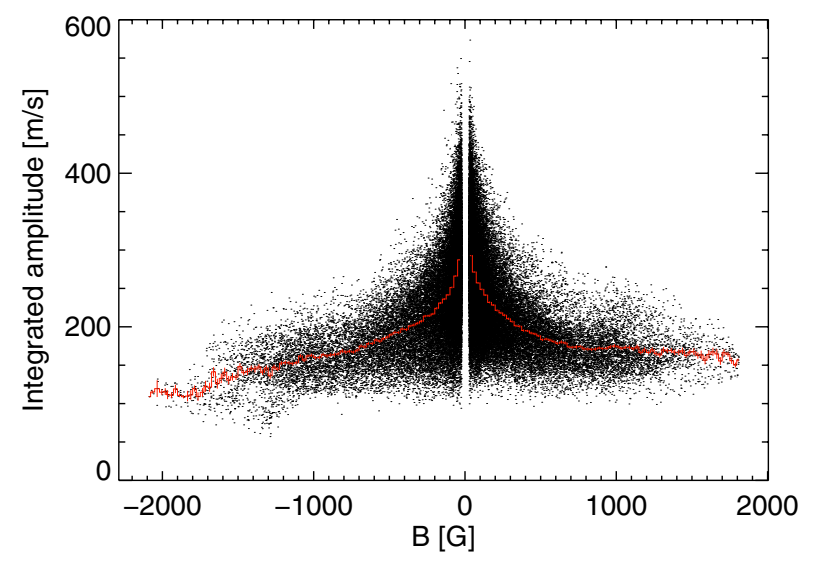

(a) AR 11166

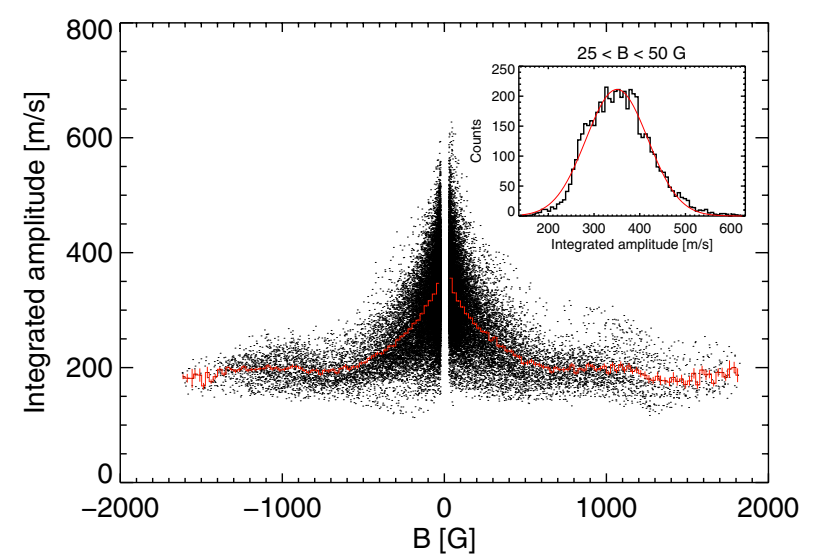

(b) AR 11316

Fig. 2. In panel a) the integrated velocity oscillation amplitude for AR11166 is shown; the same for AR1136 in panel b). The red overplots correspond to the average in each magnetic field bin. In the inset of panel b), amplitude distribution in the $25<B<50 \mathrm{G}$ bin (black line) and Gaussian fit (red line) are shown.

with each $\beta \mathrm{AR}$ and even with the polarity in the same $\beta \mathrm{AR}$, and we are well above the $20 \mathrm{~m} \mathrm{~s}^{-1}$ error on the velocity measurements due to the HMI filter transmission profiles (Fleck et al. 2011). It seems that the oscillation amplitudes are not completely suppressed, even in the strongest magnetic fields, and are not dependent on the magnetic field strength anymore. In contrast to the simulations of Cattaneo et al. (2003), it may indicate a saturation effect of the kinetic energy in the strong field regime and shed new light on the mechanisms that co-operate in the reduction of acoustic power in the lower atmosphere.

\subsection{The leading-trailing asymmetry}

To investigate the relations between local oscillation amplitude and magnetic field strength, we compute the midpoints of the amplitude profiles. Specifically, we draw horizontal lines at constant $A_{B}$ values, proceeding down in steps of $10 \mathrm{~m} \mathrm{~s}^{-1}$. We compute the abscissa of each midpoint on the segment that intersects the amplitude profiles. If these profiles were symmetric, the midpoints should be on the $B=0$ vertical line. In order to compare the amplitude profiles from different $\beta$ ARs, we normalize them to their average value $A_{B}$ in the $B= \pm 25 \mathrm{G}$ bins. For solar cycle 24 , the leading polarities are negative in the $\mathrm{N}$ hemisphere and positive in the $\mathrm{S}$ hemisphere. Figure 3a shows
Table 1. Details of SDO-HMI data used.

\begin{tabular}{|c|c|c|c|}
\hline$\beta \mathrm{AR}$ number & Observation date & Location & $S_{l} / S_{t}$ \\
\hline AR11166 & 2011.03 .08 & $\begin{array}{c}\text { N11 W01 } \\
\text { T11:00-14:00 }\end{array}$ & 0.88 \\
\hline AR11283 & 2011.09 .05 & $\begin{array}{c}\text { N13 W01 } \\
\text { T17:00-20:00 }\end{array}$ & 0.86 \\
\hline AR11302 & 2011.09 .28 & $\begin{array}{c}\text { N13 W06 } \\
\text { T17:30-20:30 }\end{array}$ & 0.96 \\
\hline AR11319 & 2011.10 .15 & $\begin{array}{c}\text { N09 E02 } \\
\text { T07:00-10:00 }\end{array}$ & 0.95 \\
\hline AR11316 & 2011.10.15 & $\begin{array}{c}\text { S12 E01 } \\
\text { T07:00-10:00 }\end{array}$ & 0.90 \\
\hline AR11330 & 2011.10 .27 & $\begin{array}{c}\text { N09 E04 } \\
\text { T18:30-21:30 }\end{array}$ & 0.83 \\
\hline AR11338 & 2011.11.06 & $\begin{array}{c}\text { S14 W00 } \\
\text { T20:00-23:00 }\end{array}$ & 0.92 \\
\hline AR11341 & 2011.11.11 & $\begin{array}{c}\text { N08 W00 } \\
\text { T07:00-10:00 }\end{array}$ & 1.05 \\
\hline AR11362 & 2011.12.03 & $\begin{array}{c}\text { N08 W06 } \\
\text { T20:00-23:00 }\end{array}$ & 1.10 \\
\hline AR11367 & 2011.12 .07 & $\begin{array}{c}\text { S18 W05 } \\
\text { T20:00-23:00 }\end{array}$ & 0.90 \\
\hline AR11375 & 2011.12 .13 & $\begin{array}{c}\text { N08 W03 } \\
\text { T19:00-22:00 }\end{array}$ & 0.82 \\
\hline AR11389 & 2012.01 .03 & $\begin{array}{c}\text { S22 W05 } \\
\text { T16:00-19:00 }\end{array}$ & 0.97 \\
\hline
\end{tabular}

Notes. The fourth column shows the leading to trailing polarity area ratio.

the midpoint lines for all the $12 \beta$ ARs considered in this study. Northern and southern $\beta \mathrm{AR}$ families appear well defined and separated. Moreover, as can be seen in Fig. 3, the amount of asymmetry, defined as the distance of each midpoint from the vertical line through $B=0$, increases for stronger fields. In the $\mathrm{N}$ hemisphere (in blue), it extends up to $|\mathbf{B}| \simeq 230 \mathrm{G}$, while in $\mathrm{S}$ hemisphere (in red), it is less apparent, and only reaches $|\mathbf{B}| \simeq 130 \mathrm{G}$. The amplitude profiles of $\mathrm{N} \beta \mathrm{ARs}$ are biased toward positive polarities, i.e., the trailing, while the $\mathrm{S} \beta \mathrm{ARs}$ are biased toward negative polarities, i.e., the trailing again.

To visualize the asymmetry differently, we can fix a value $|\mathbf{B}|$ for the magnetic strength and compute the amplitude difference, i.e., the difference between the respective averaged amplitudes for both polarities in each $\beta \mathrm{AR}$, namely, $A_{|\mathbf{B}|}-A_{-|\mathbf{B}|}$. In Fig. 3b, we show these differences vs the absolute value of magnetic strength for all $\beta$ ARs of Table 1 . This plot confirms that the asymmetry is lower where the field is weaker. At $25 \mathrm{G}$ the difference is about $10 \mathrm{~m} \mathrm{~s}^{-1}$ and typical values for velocity amplitudes up to $600 \mathrm{~m} \mathrm{~s}^{-1}$; at $1000 \mathrm{G}$ the difference rises to over $50 \mathrm{~m} \mathrm{~s}^{-1}$, while the amplitudes are $\sim 200 \mathrm{~m} \mathrm{~s}^{-1}$. Both in $\mathrm{N}$ and $\mathrm{S}$ hemispheres, the oscillation amplitude in the trailing polarity is higher than that in the leading polarity. This fact implies that $A_{|\mathbf{B}|}>A_{-|\mathbf{B}|}$ in the $\mathrm{N}$ hemisphere and vice versa in the $\mathrm{S}$ hemisphere, and that the amplitude reduction is less efficient in the trailing polarity.

Such an asymmetry suggests that the amount of oscillation reduction within magnetic environments is not a function of the local magnetic field strength only. If this were the case, we would observe the same oscillation amplitudes for $\mathbf{B}$ and $-\mathbf{B}$ magnetic fields. This would imply that both plots of Fig. 3 lie on the line through $0 \mathrm{G}$ (a) and $0 \mathrm{~m} \mathrm{~s}^{-1}$ (b), respectively.

The simulations of Fan et al. (1993) about area asymmetries of $\beta$ ARs predicted a pronounced difference in field strength between the leading and the trailing polarities, as well as ensuing 


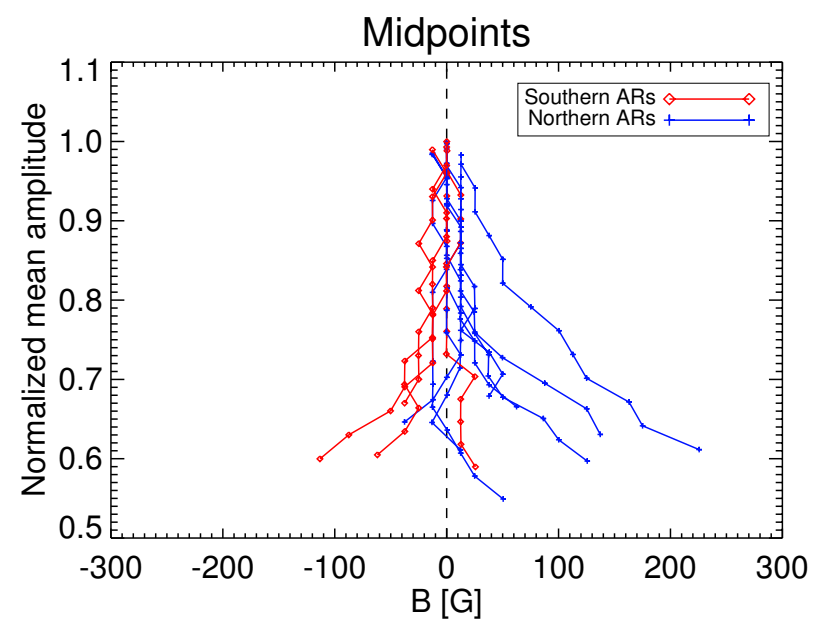

(a)

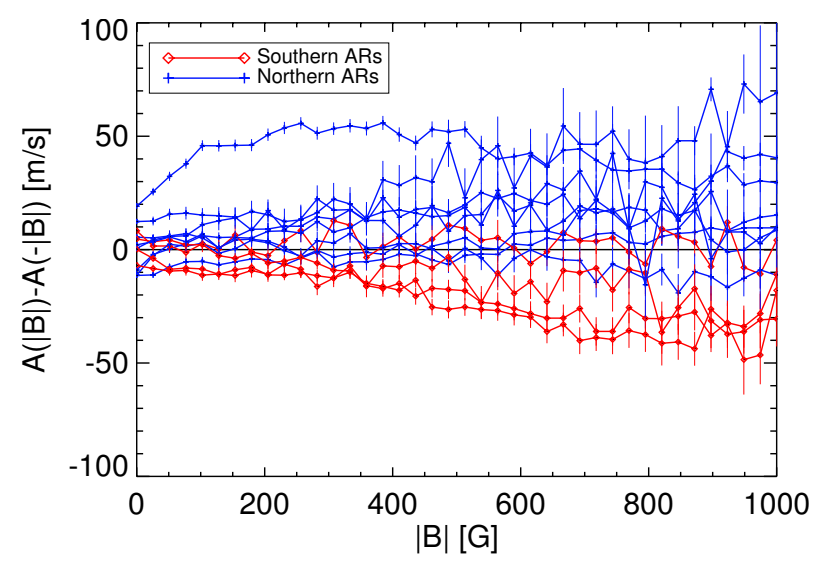

(b)

Fig. 3. a) Midpoints for normalized velocity oscillation amplitude. b) Difference between oscillation amplitude for both polarities against the magnetic strength. Bars represent errors. Red line with diamonds refers to $\mathrm{S} \beta \mathrm{ARs}$, blue line with plus signs to $\mathrm{N} \beta$ ARs.

differences in their fragmentation and lifetimes. This suggests that the asymmetry we found could be derived from nonlocal properties, i.e., on the whole magnetic environment topology and even its surroundings.

We therefore computed the area asymmetry in our magnetograms following Yamamoto (2012), with a threshold $B>$ $500 \mathrm{G}$. The results are reported in the fourth column of Table 1. We found that, on average, the area of the leading polarity is $\sim 90 \%$ of the trailing polarity area, regardless of the hemisphere which the $\beta$ AR belongs to. This quantifies how much a trailing polarity is spread with respect to its leading polarity. We can speculate that a relation may exist between the area and the oscillation suppression due to a different interaction of acoustic waves with sparser or denser active region polarities.

\subsection{Oscillation amplitude vs. magnetic field inclination The case of AR11166}

To support the findings reported in Sect. 4.2, we studied the dependence of oscillation amplitude on the magnetic field inclination angle $\theta$ in an $\beta \mathrm{AR}$. For this purpose, we considered the AR11166 (Fig. 1a) as a case study. We used the line-ofsight (LOS) inclination maps provided by the VFISV (Very Fast

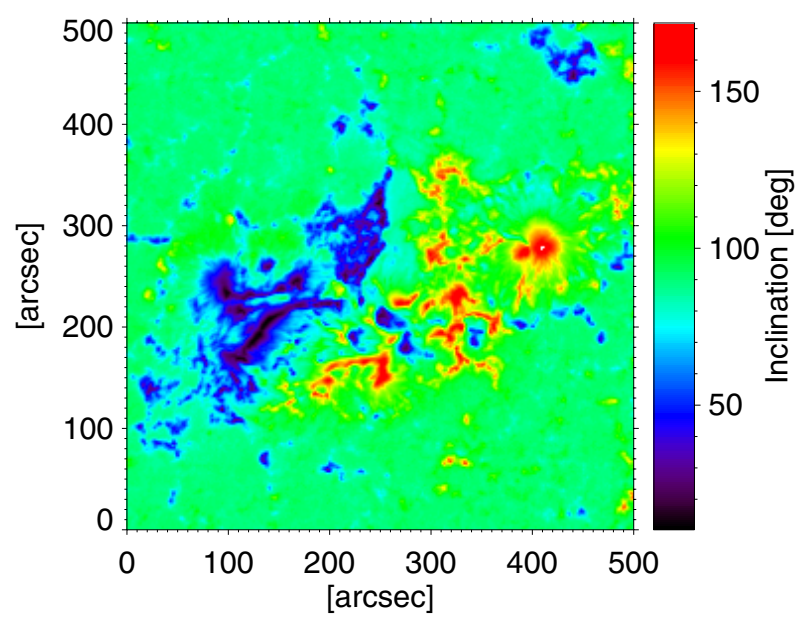

Fig. 4. Inclination map of AR 11166. The leading polarity (negative) ranges from $90^{\circ}$ to $180^{\circ}$ and the trailing polarity (positive) from $0^{\circ}$ to $90^{\circ}$.

Inversion of the Stokes Vector, Borrero et al. 2011) inversion of HMI vector magnetic field data.

In Fig. 4 we show the magnetic field inclination map of AR11166. The inclinations retrieved by the VFISV span the range $10^{\circ}<\theta<172^{\circ}$. Following the most-used sign convention, the leading polarity (negative) ranges from $90^{\circ}$ to $180^{\circ}$ and the trailing polarity (positive) from $0^{\circ}$ to $90^{\circ}$. As expected, the innermost umbrae are mostly line of sight, as the $\beta$ ARs were selected as close as possible to the disk center.

VFISV also provides the error $\sigma_{\theta}$ associated to the inclination for each pixel (Borrero et al. 2011; Press et al. 1986). To avoid polarity flips due to the inversion error, we discarded all those pixels where $\theta \pm 3 \sigma_{\theta}$ causes the field to flip its inclination from positive to negative (or vice versa). We stress that VFISV is a Milne-Eddington code and is therefore blind to any $B$ and $v$ LOS gradients, which produce asymmetries in the Stokes profiles. This happens most often in regions filled by weak fields (Viticchié et al. 2011; Viticchié \& Sánchez Almeida 2011). Moreover, the inversion of low signal-to-noise Stokes profiles is usually problematic (see Landi Degl'Innocenti 1992; Borrero et al. 2011, for more details). For noisy Stokes profiles and therefore low $B$ values, VFISV (and any inversion code) tends to be biased toward $\sim 90^{\circ}$ inclinations (Borrero \& Kobel 2011). For these reasons and for consistency with the threshold used in Sect. 3, we rejected the pixels with $B<25 \mathrm{G}$ and $75^{\circ}<\theta<105^{\circ}$. After this selection, about $17 \%$ of the initial pixels were selected for the analysis.

We plotted $A_{x y}$ and its average in each $\theta$ bin $A_{\theta}=\left\langle A_{x y}\right\rangle_{\theta}$ against the magnetic field inclination $\theta$ (shown in Fig. 5). For ease of comparison, in the upper panel of Fig. 6 we show again $A_{\theta}$ versus $\theta$ in the range $\left[10^{\circ}, 75^{\circ}\right]$. Both $A_{\theta}$ smoothly decrease from $\simeq 250 \mathrm{~m} \mathrm{~s}^{-1}$ to $\sim 140 \mathrm{~m} \mathrm{~s}^{-1}$, from almost horizontal to almost vertical fields, respectively, but the oscillation amplitude reduction is more effective for the leading polarity (red curve) than for the trailing polarity (blue curve).

In the lower panel of Fig. 6 we plot the oscillation amplitude asymmetry $\Delta A=A_{\theta}^{T}-A_{\theta}^{L}$ VS $\theta$. Within the errors up to $\theta \simeq$ $45^{\circ} \Delta A$ is constant $\left(\simeq 15 \mathrm{~m} \mathrm{~s}^{-1}\right)$, but then drops to very few $\mathrm{m} \mathrm{s}^{-1}$.

The inclination analysis shows that $\mathrm{a} \simeq 15 \mathrm{~m} \mathrm{~s}^{-1}$ asymmetry exists between the polarities of AR11166. In particular, the velocity oscillation amplitude is enhanced in the trailing polarity with respect to the leading polarity. 
F. Giannattasio et al.: On the asymmetry of velocity oscillation amplitude in bipolar active regions

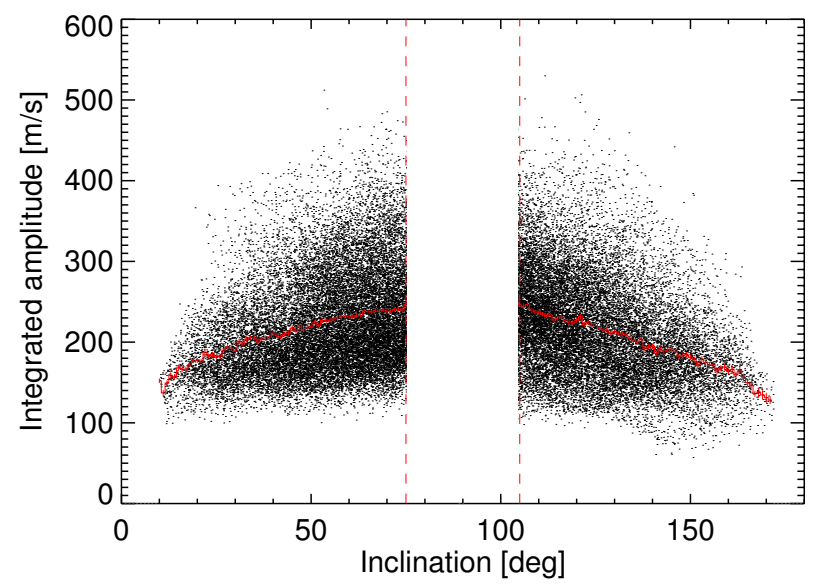

Fig. 5. Integrated velocity oscillation amplitude within AR11166 as a function of the magnetic field inclination $\theta$. The thick red line corresponds to the average amplitude $A_{\theta}=\left\langle A_{x y}\right\rangle_{\theta}$ in each $\theta$ bin $\left(\Delta \theta=1^{\circ}\right)$. The vertical dashed lines mark the threshold $\theta<75^{\circ}$ OR $\theta>105^{\circ}$.

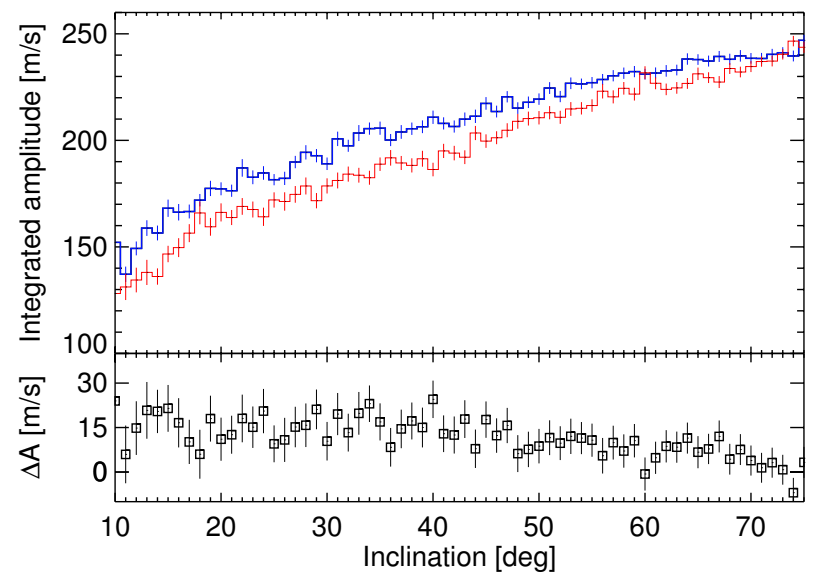

Fig. 6. Upper panel: $A_{\theta}^{T}$ and $A_{\theta}^{L}$ versus $\theta$ in the range $\left[10^{\circ}, 75^{\circ}\right]$. The blue line represents the average amplitude of the positive (trailing) polarity $A_{\theta}^{T}$. The red line represents the average amplitude of the negative (leading) polarity after a remapping operation $\left(\theta \rightarrow 180^{\circ}-\theta: A_{\theta}^{L}\right)$. Lower panel: $\Delta A$ versus $\theta$ (see the text). Vertical bars represent the errors, which were computed for each $A_{\theta}$ with the same method described in Sect. 3 and then summed up.

There is no evident relation between the asymmetry $\Delta A$ and the inclination $\theta$. For any $\theta \lesssim 45^{\circ}$ the oscillation amplitude asymmetry is positive and around $15 \mathrm{~m} \mathrm{~s}^{-1}$. We interpret the drop at $\simeq 45^{\circ}$ as the combined effect of the VFISV preference to associate weak fields with larger inclinations (Borrero \& Kobel 2011) and the amplitude difference, which is small for weak fields (see Fig. 3b). Also, an incorrectly retrieved weak $\mathbf{B}$ may result from unresolved magnetic structuring in the pixel (e.g., Sánchez Almeida 1998; Socas-Navarro \& Sánchez Almeida 2003; Viticchié et al. 2011), and we recall that a VFISV hypothesis is that the magnetic field is constant within the pixel.

Schunker \& Cally (2006) and Stangalini et al. (2011) have demonstrated that, due to mode conversion, there exists a preferred angle at which the power is significant larger. In our case, we focus on the amplitude asymmetry between the two polarities instead of considering the proper amplitude dependence, which may be affected by biases due to the magnetic field strength.

\section{Conclusions}

In this work, we focused on the velocity oscillation amplitude reduction in $\beta \mathrm{ARs}$, reaching the following conclusions. The oscillation amplitude reduction found in magnetic environments of $\beta$ ARs is $0.54 \pm 0.06$, which agrees with the values quoted in the literature (e.g. Woods \& Cram 1981; Lites et al. 1982; Braun et al. 1987; Tarbell et al. 1988). In addition, the five-minute amplitudes are not completely suppressed in the strongest magnetic fields of the spots' innermost umbra. It would be very important to understand why a plateau appears in the oscillation amplitude profiles for strong fields.

There exists a leading-trailing polarity asymmetry in $\beta$ ARs. The asymmetry suggests that the reduction in oscillation amplitude does not depend on the field strength only, but also depends on nonlocal conditions, such as the area on which the field spreads. Furthermore, the trailing polarity systematically shows a higher oscillation amplitude than the leading polarity, regardless of the hemisphere which the $\beta$ AR belongs to.

The plot of the velocity oscillation amplitude as a function of the magnetic field inclination confirms such an asymmetry in $\beta$ ARs. Apparently, the asymmetry does not depend on the inclination.

We used HMI full-disk data at a 45 s time cadence and at 1 arcsec spatial resolution. An analysis of these data revealed a possible saturation of the oscillation amplitude reduction for strong $B$ and an asymmetry in such a reduction for leadingtrailing polarities. These results have to be accounted for in modeling the power reduction in magnetic environments, as well as in the emergence and the evolution of $\beta$ ARs.

Acknowledgements. We thank Prof. Stuart M. Jefferies (IfA, University of Hawaii) for the useful discussion and critical reading of the early version of this manuscript. SDO-HMI data are courtesy of the NASA/SDO HMI science team. We acknowledge the VSO project (http://vso.nso.edu) through which data were easily obtained.

\section{References}

Abdelatif, T. E., Lites, B. W., \& Thomas, J. H. 1986, ApJ, 311, 1015 Balthasar, H., \& Woehl, H. 1980, A\&A, 92, 111

Bogdan, T. J., Brown, T. M., Lites, B. W., \& Thomas, J. H. 1993, ApJ, 406, 723 Bogdan, T. J., Hindman, B. W., Cally, P. S., \& Charbonneau, P. 1996, ApJ, 465, 406

Borrero, J. M., \& Kobel, P. 2011, A\&A, 527, A29

Borrero, J., Tomczyk, S., Kubo, M., et al. 2011, Sol. Phys., 273, 267

Braun, D. C., Duvall, Jr., T. L., \& Labonte, B. J. 1987, ApJ, 319, 27

Braun, D. C., Duvall, Jr., T. L., \& Labonte, B. J. 1988, ApJ, 335, 1015

Braun, D. C., Lindsey, C., Fan, Y., \& Jefferies, S. M. 1992, ApJ, 392, 739

Bray, R. J., \& Loughhead, R. E. 1979, Sunspots

Brown, T. M., Bogdan, T. J., Lites, B. W., \& Thomas, J. H. 1992, ApJ, 394, L65

Cally, P. S. 1995, ApJ, 451, 372

Cally, P. S., \& Andries, J. 2010, Sol. Phys., 266, 17

Cally, P. S., \& Bogdan, T. J. 1993, ApJ, 402, 721

Cally, P. S., \& Bogdan, T. J. 1997, ApJ, 486, 67

Cally, P. S., Crouch, A. D., \& Braun, D. C. 2003, MNRAS, 346, 381

Cameron, R., Gizon, L., \& Duvall, Jr., T. L. 2008, Sol. Phys., 251, 291

Cattaneo, F., Emonet, T., \& Weiss, N. 2003, ApJ, 588, 1183

Crouch, A. D., \& Cally, P. S. 2003, Sol. Phys., 214, 201

Fan, Y., Fisher, G. H., \& Deluca, E. E. 1993, ApJ, 405, 390

Fleck, B., Couvidat, S., \& Straus, T. 2011, Sol. Phys., 271, 27

Gordovskyy, M., \& Jain, R. 2008, ApJ, 681, 664

Hindman, B. W., \& Brown, T. M. 1998, ApJ, 504, 1029

Hindman, B. W., \& Jain, R. 2008, ApJ, 677, 769

Hindman, B. W., \& Jain, R. 2012, ApJ, 746, 66

Hindman, B. W., Jain, R., \& Zweibel, E. G. 1997, ApJ, 476, 392

Jain, R., Hindman, B. W., \& Zweibel, E. G. 1996, ApJ, 464, 476

Jain, R., Hindman, B. W., Braun, D. C., \& Birch, A. C. 2009, ApJ, 695, 325

Landi Degl'Innocenti, E. 1992, Magnetic field measurements, eds. F. Sanchez,

M. Collados, \& M. Vazquez, 71

Leighton, R. B., Noyes, R. W., \& Simon, G. W. 1962, ApJ, 135, 474 
A\&A 550, A47 (2013)

Lites, B. W., White, O. R. \& Packman, D. 1982, ApJ, 253, 386

Moreno-Insertis, F., Caligari, P., \& Schuessler, M. 1994, Sol. Phys., 153, 449

Parchevsky, K. V., \& Kosovichev, A. G. 2007, ApJ, 666, L53

Petrovay, K., Marik, M., Brown, J. C., Fletcher, L., \& van Driel-Gesztelyi, L. 1990, Sol. Phys., 127, 51

Pradeep Chitta, L., Jain, R., Kariyappa, R., \& Jefferies, S. M. 2012, ApJ, 744, 98 Press, W. H., Flannery, B. P., \& Teukolsky, S. A. 1986, Numerical recipes The art of scientific computing

Roberts, B., \& Webb, A. R. 1978, Sol. Phys., 56, 5

Sánchez Almeida, J. 1998, in Three-Dimensional Structure of Solar Active Regions, eds. C. E. Alissandrakis, \& B. Schmieder, ASP Conf. Ser., 155, 54

Scherrer, P., Schou, J., Bush, R., et al. 2012, Sol. Phys., 275, 207

Schou, J., Scherrer, P., Bush, R., et al. 2012, Sol. Phys., 275, 229

Schunker, H., \& Cally, P. S. 2006, MNRAS, 372, 551

Socas-Navarro, H., \& Sánchez Almeida, J. 2003, ApJ, 593, 581

Spruit, H. C. 1981, A\&A, 98, 155
Spruit, H. C., \& Bogdan, T. J. 1992, ApJ, 391, 109

Stangalini, M., Del Moro, D., Berrilli, F., \& Jefferies, S. M. 2011, A\&A, 534, A65

Stangalini, M., Giannattasio, F., Del Moro, D., \& Berrilli, F. 2012, A\&A, 539, L4

Tarbell, T. D., Peri, M., Frank, Z., Shine, R., \& Title, A. M. 1988, in ESA Spec. Publ. 286, Seismology of the Sun and Sun-Like Stars, ed. E. J. Rolfe, 315

Ternullo, M., Zappala, R. A., \& Zuccarello, F. 1981, Sol. Phys., 74, 111

Title, A. M., Topka, K. P., Tarbell, T. D., et al. 1992, ApJ, 393, 782

Toner, C. G., \& Labonte, B. J. 1993, ApJ, 415, 847

van Driel-Gesztelyi, L., \& Petrovay, K. 1990, Sol. Phys., 126, 285

Viticchié, B., \& Sánchez Almeida, J. 2011, A\&A, 530, A14

Viticchié, B., Sánchez Almeida, J., Del Moro, D., \& Berrilli, F. 2011, A\&A, 526, A60

Woods, D. T., \& Cram, L. E. 1981, Sol. Phys., 69, 233

Yamamoto, T. T. 2012, A\&A, 539, A13

Zwaan, C. 1985, Sol. Phys., 100, 397 